\title{
Short Column Advantage Justified by Information Theory of Optimization in Chromatography
}

\author{
Yuzuru HaYaShI and Rieko Matsuda \\ National Institute of Hygienic Sciences, Kamiyoga, Setagaya, Tokyo 158, Japan
}

Keywords Optimization, chromatography, information theory, Kalman filter, FUMI

A major aim in quantitative analysis is to obtain the highest possible precision of measurements through analytical means within a satisfactorily short observation time. Massart et al. stated that precision and accuracy are the first to come to mind in selecting a developed analytical procedure. ${ }^{1}$ When an analytical system is evaluated, the extent to which its aim has been achieved should be a major reference.

In column chromatography, methods have so far often been evaluated by criteria based on the chromatographic resolution $R_{\mathrm{s}} .{ }^{2-5}$ However, the separation of peaks is a means for precise analysis, although it is among the most influential factors regarding precision; $\boldsymbol{R}_{\mathrm{s}}$-based criteria are functions describing the extent to which the means has been accomplished in chromatography. Such a description of the means would not reach fair judgment of analytical systems.

A recently developed function called FUMI (Function of Mutual Information) has succeeded in explicitly describing analytical precision as the Shannon mutual information. ${ }^{6}$ The superiority of methods, therefore, can be judged according to FUMI. FUMI is derived from information theory ${ }^{7}$ and one-dimensional Kalman filter theory. ${ }^{8,9}$ The judgment on FUMI allows us to obtain new knowledges concerning chromatographic analysis which cannot be predicted by $\boldsymbol{R}_{\mathbf{s}}$. A typical example is in the title.

\section{Theory}

FUMI $\phi_{j}$ for peak $j$ takes the form: $\phi_{j}=-\log \left(\mathrm{S}_{j}\right)(\geq 0)$ where $S_{j}$ denotes the precision of measurements (the standard deviation of errors; the mean is zero). ${ }^{10,11} \phi_{j}$ can also be given as the subtraction of the information loss $\delta \phi_{j}(\geq 0)$ arising from peak overlap from the intact information $\psi_{j}(\geq 0):{ }^{12}$

$$
\phi_{j}=\psi_{j}-\delta \phi_{j}
$$

where $\psi_{j}$ is independent of the peak overlap and denotes the information indigenous to the whole peak shape, itself. For Gaussian peak $j$ of area $A_{j}$, retention time $\tau_{j}$ and width $\sigma_{j}$, the intact information is described by ${ }^{13}$

$$
\psi_{j}=\frac{1}{2} \log \left(\frac{A_{j}^{2} N^{1 / 2}}{2 \pi^{1 / 2} \tau_{j} \tilde{a}}\right)
$$

where $N$ denotes the plate number $\left(=\tau_{j}^{2} / \sigma_{j}^{2}\right)$; constant $\widetilde{a}$ is the power spectrum intensity of the white noise in a chromatogram. Of central importance in this paper is the different informational contributions of the variables $A_{j}, \tau_{j}, \sigma_{j}$ and $N$.

The most prominent differences of FUMI from the other quality criteria are: $\phi_{j}$ is defined for a single peak (clearly, even a peak can transmit useful information); the total information $\Phi$ for a multi $(q)$-peak system takes the linear sum: $\Phi=\sum_{j=1}^{q} \phi_{j}$.

\section{Informational effects of column length and flow rate}

The problem treated here is how we can obtain more information from a chromatogram by manipulating only the column length $L$ and flow rate $v$.

Figure 1 (a) shows a chromatogram (simulation) of two peaks sufficiently separated $(\Phi=16.01, \delta \Phi=0(=$ $\left.\left.\sum_{j=1}^{q} \delta \phi_{j}\right), R_{s}=2.38\right)$. The column shortening $(L / 2)$ causes less separation $\left(R_{s}=1.68\right)$ but more information $(\Phi=16.36)$ (see Fig. 1(b)), mainly because of the peak sharpness gained by the procedure. For further information, $v$ is halved (see Fig. 1(c)), increasing the area $\left(2 A_{j}\right)$ and generating much more information $(\Phi=17.05) ; R_{s}$ is invariant in this step $\left(R_{s}=1.68\right)$. From these chromatograms, we can see that chromatogram (c), in spite of inferior column efficiency $N$ and poor separation, can provide much more information than (a).

The difference in $\Phi$ of the chromatograms in question can be easily calculated from Eq. (2); note $\delta \phi_{j}=0$ and $\phi_{j}=\psi_{j}$. The variables are: (a) $A_{j}, \tau_{j}$ and $N$; (b) $A_{j}, \tau_{j} / 2$ and $N / 2$; (c) $2 A_{j}, \tau_{j}$ and $N / 2$. Substituting these variables in to Eq. (2), we can obtain the change in the information; e.g. $\Phi(\mathrm{c})-\Phi(\mathrm{a})=\log 2^{2}-\log 2^{1 / 2} \approx 1$. This informational increase is mainly because $A_{j}$ is the most 


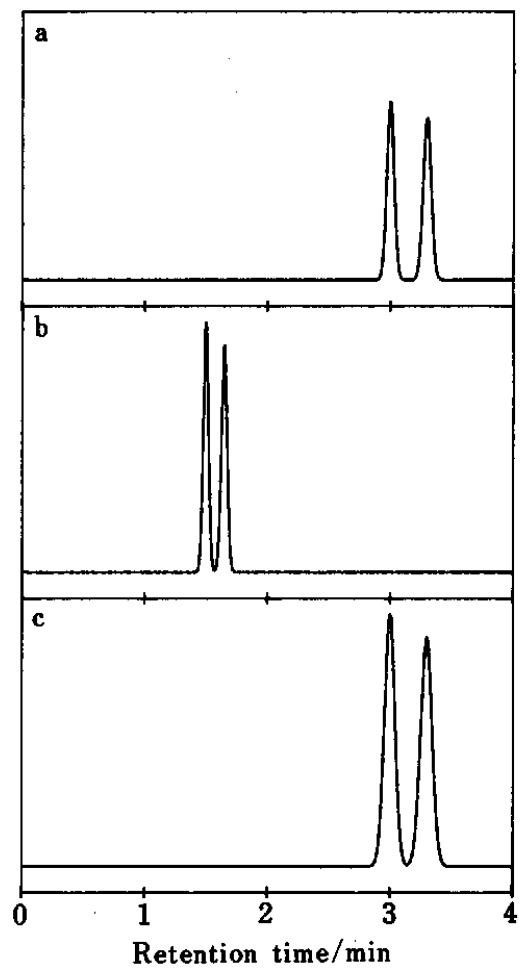

Fig. 1 Chromatograms ( $\delta \Phi=0$ ) in (a) $L$ and $\nu$, (b) $L / 2$ and $v$ and (c) $L / 2$ and $v / 2$. Initial conditions (a): $A_{j}=10000$; $N=10000 ; \tau_{1}=3 \mathrm{~min} ; \tau_{2}=3.3 \mathrm{~min} ; \tilde{a}=1$.

influential factor on FUMI, rather than $\tau_{j}$ or $N$ (compare the exponents of Eq. (2)).

If the column length $L$ is doubled in the above situation, the information decreases due to the long retention $2 \tau_{j}$ and the wide peak $2^{1 / 2} \sigma_{j}$, despite the increases in $N$ and $R_{s}\left(2 N\right.$ and $\left.2^{1 / 2} R_{s}\right)$. The difference in FUMI $\phi_{j}$ is $-\left(\log 2^{1 / 2}-\log 2\right) \approx 0.35$.

The improvement in the column efficiency $N$, itself, has the disparate views from the column elongation resulting in the improvement in $N$. If $N$ is doubled without column elongation, then the intact information $\psi_{j}$ of every peak increases by $(1 / 4) \log 2\left(=(1 / 2) \log 2^{1 / 2}\right)$. If $L$ is doubled, every $\psi_{j}$ decreases by $(1 / 4) \log 2(=-[(1 / 2)$ $\left.\left.\times \log 2^{1 / 2}-(1 / 2) \log 2\right]\right)$. The improvement in column packing always exerts the favorable effects on the information $\psi_{j}$ inherent to peak shape. On the contrary, the column elongation adversely affects the information $\psi_{j}$ in any case, although it can lead to the increase in the mutual information $\phi_{j}$ through the relaxation of strong peak overlap. Column length attenuates the information; the ideal column is of infinite $N$ and of infinitesimal $L$.

It might have often been proposed so far that even the chromatogram (c) can satisfy modest requirements of analysts, but until the development of FUMI, it cannot be proved theoretically that (c) of a large $\Phi$ is superior to the seemingly "good" (a) indicated by a large $R_{\mathrm{s}}$ and $N$. This conclusion also gives a theoretical

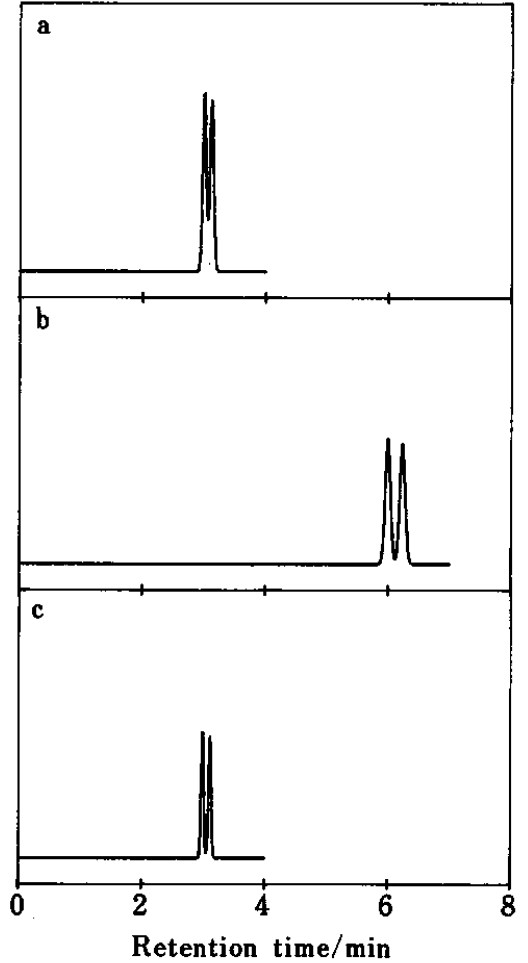

Fig. 2 Chromatograms in (a) $L$ and $v$, (b) $2 L$ and $v$ and (c) $2 L$ and $2 v$. Initial conditions (a): $A_{j}=10000 ; N=10000 ; \tau_{1}=3 \mathrm{~min}$; $\tau_{2}=3.12 \mathrm{~min} ; \tilde{a}=1$.

evidence for a common view that an excess separation of peaks degrades chromatographic performance.

Not every peak pair of an $R_{s}$ value can realize the informational effect mentioned above. Figure 2(a) shows strongly overlapped peaks of $\Phi=15.65$ and $\delta \Phi=0.39\left(R_{s}=0.98\right)$. The same procedures as Fig. 1 induce the fatal reduction in FUMI $(\Phi=6.72)$. A common strategy is effective for an analytical improvement in this condition (see below), but $R_{s}$ cannot follow the informational behaviors in this case.

In the first place, the large $\delta \Phi$ should be eliminated. Unlike the situation of Fig. 1, $N$ plays the most important role in FUMI, rather than $A_{j}$ or $\tau_{j}$. The chromatogram obtained from a column of twice the length is shown in Fig. 2(b) $\left(\Phi=15.69, \delta \Phi=0.00, R_{\mathrm{s}}=1.39\right)$. The unexpectedly small increase in the information $(\Phi(\mathrm{b})-$ $\Phi(a)=0.04)$ comes from an excessive peak widening and a decrease in $\psi_{j}$. The doubled flow rate $2 v$ causes the halved area $A_{j} / 2$ and reduction of FUMI ( $\Phi=15.00$, $\delta \Phi=0.00, R_{\mathrm{s}}=1.39$ ) (see Fig. 2(c)).

Our conclusion is clear from the viewpoint of information theory. Yet, it may be hard to comfirm it by experiments, because the sample-injection error will remain dominant among the total error sources in a real system, and is not involved in FUMI. The chromatographic model on which FUMI is founded comprises the errors arising from peak overlap and the noise level. ${ }^{14}$ The injection error is theoretically trivial 
in chromatography and ignorance of it does not disparage FUMI in either theory or practice.

FUMI enables us to select the optimal chromatogram among all the experimental conditions examined. ${ }^{10-16}$ All the chromatographic variables which can be related to $A_{j}, \sigma_{j}$ and $\tau_{j}$ can be optimized by FUMI and that, simultaneously; e.g., detection wavelength ${ }^{14}$, the amount of internal standard. ${ }^{16}$ FUMI would establish a new direction of chromatographic analyses.

The authors thank Dr. A. Nakamura and Dr. S. Kojima (National Institute of Hygienic Sciences) for useful discussion.

\section{References}

1. D. L. Massart, B. G. M. Vandeginste, S. N. Deming, Y. Michotte and L. Kaufman, "Chemometrics: a textbook", Elsevier, Amsterdam, 1988.

2. H. J. G. Debets, B. L. Bajema and D. A. Doornbos, Anal. Chim. Acta, 151, 131 (1983).

3. C. E. Goewie, J. Liq. Chromatogr., 9, 1431 (1985).

4. P. J. Schoenmakers, J. Liq. Chromatogr., 10, 1865 (1987).
5. J. L. Glajch, J. J. Kirkland and J. M. Minor, J. Liq. Chromatogr., 10, 1727 (1987).

6. Y. Hayashi, S. Yoshioka and Y. Takeda, Anal. Sci., 5, 329 (1989).

7. S. Arimoto, "Kalman Filter", Sangyo Tosho, Tokyo, 1977.

8. Y. Hayashi, S. Yoshioka and Y. Takeda, Anal. Chim. Acta, 212, 81 (1988).

9. Y. Hayashi, R. Matsuda, S. Yoshioka and Y. Takeda, Anal. Chim. Acta, 209, 45 (1988).

10. R. Matsuda, Y. Hayashi, M. Ishibashi and Y. Takeda, J. Chromatogr., 462, 13 (1989).

11. R. Matsuda, Y. Hayashi, M. Ishibashi and Y. Takeda, J. Chromatogr., 462, 23 (1989).

12. Y. Hayashi and R. Matsuda, Anal. Chim. Acta, 222, 313 (1989).

13. Y. Hayashi, Anal. Sci., 6, 15 (1990).

14. Y. Hayashi and R. Matsuda, Anal. Sci., 5, 459 (1989).

15. R. Matsuda, Y. Hayashi, M. Ishibashi and Y. Takeda, Anal. Chim. Acta, 222, 301 (1989).

16. Y. Hayashi and R. Matsuda, submitted for publication.

(Received August 25, 1989) (Accepted November 14, 1989) 